\title{
Calcofluor White Alters the Assembly of Chitin Fibrils in Saccharomyces cerevisiae and Candida albicans Cells
}

\author{
By M. VICTORIA ELORZA, HORTENSIA RICO AND \\ RAFAEL SENTANDREU* \\ Departamento de Microbiología, Facultad de Farmacia, Universidad de Valencia, Avda. Blasco \\ Ibañez 13, Valencia-10, Spain
}

(Received 3 November 1982)

\begin{abstract}
In the presence of calcofluor white, budding scars and dividing cross-walls of Saccharomyces cerevisiae exhibited fluorescence, indicating that the brightener was a specific marker of fungal chitin. In addition, incubation of cells in the presence of the brightener did not stop protein and wall-polymer formation, but abnormal deposition of chitin occurred. Chitin synthesis was normal in regenerating protoplasts of Candida albicans in the presence of calcofluor, but formation of the crystalline lattice was blocked. These results suggest that crystallization of nascent subunits may occur by a self-assembly mechanism that was blocked by the stain.
\end{abstract}

\section{INTRODUCTION}

Cell wall formation is the result of two processes: (i) synthesis and secretion of the polymers involved, and (ii) interaction and assembly of these polymers outside the cytoplasm to form the wall. If any of these processes is modified or blocked, changes in the spatial organization of the wall should be expected. Accordingly, a temperature-sensitive secretory mutant of Saccharomyces cerevisiae that accumulated intracellular precursor forms of exported proteins showed alteration in cell morphology (Novick et al., 1981), while in those mutants defective in gene $c d c 24$ an abnormal deposition of chitin (Sloat \& Pringle, 1978) and mannan (Sloat et al., 1981) took place.

Less is known about the mechanisms involved in assembly of wall components once they have been released from the plasma membrane into the periplasmic space. A self-assembly mechanism of subunits by physicochemical interactions was suggested as a mechanism of $\beta$ glucan crystallization (Eddy \& Woodhead, 1968), and chitin microfibrils have been obtained from isolated chitin synthase preparations (Duran \& Cabib, 1978; Ruiz-Herrera et al., 1975).

Calcofluor is a fluorescent brightener that has been used to whiten textiles and paper because of its ability to hydrogen-bond to cellulose. In addition, this compound has also been used in the topological location of cellulose (Haigler et al., 1980) and chitin (Herth, 1980).

One of the key difficulties in a study of microfibril formation in intact cells is the presence of the pre-existing wall which masks the process. In order to overcome this difficulty, formation of fibrils may be examined in protoplasts, which offer a valuable experimental system for analysing fibril formation. In protoplasts, the interaction between nascent (fibrillar and matrix) wall polymers that takes place in growing cells is initially absent or lowered because of the release of matrix polymers into the surrounding medium.

In the present work, calcofluor white was used to localize chitin deposition and to disrupt microfibril formation in actively growing cells of $S$. cerevisiae and protoplasts of Candida albicans. 


\section{METHODS}

Strains. The organisms used were Saccharomyces cerevisiae A364A (a adel ade 2 ura his 7 lys 2 tyr 1 gall) obtained from L. H. Hartwell, University of Washington, Seattle, and Candida albicans ATCC 26555.

Saccharomyces cerevisiae was maintained on slants of medium YM-1 and cells propagated in liquid YM-1 medium (Elorza et al., 1976). Candida albicans was maintained on slants of Sabouraud-dextrose agar and propagated in the medium of Lee et al. (1975).

Preparation of protoplasts. Protoplasts were obtained by treatment with Zymolyase $\left(2 \mathrm{mg} \mathrm{ml}^{-1}\right.$; Kirin Breweries Co., Takasaki, Japan) in $0.6 \mathrm{M}-\mathrm{KCl}$ after pretreatment in $50 \mathrm{~mm}$-dithiothreitol, $5 \mathrm{~mm}$-EDTA and $0.5 \mathrm{mg}$ pronase $\mathrm{ml}^{-1}$ for $30 \mathrm{~min}$ at $30^{\circ} \mathrm{C}$. Regeneration of protoplasts was carried out in the medium of Lee et al. (1975) supplemented with $0.6 \mathrm{M}-\mathrm{KCl}$ as an osmotic stabilizer. In some experiments, regeneration of protoplasts was carried out in the presence of $50 \mu \mathrm{g}$ calcofluor white M2R (American Cyanamid Co., Bound Brook, U.S.A.) $\mathrm{ml}^{-1}$. Concentrations of proteins and wall polymers were determined as previously described (Elorza et al., 1976).

Fluorescence microscopy. Cells were resuspended in $50 \mu \mathrm{g}$ calcofluor ( $\mathrm{ml}$ distilled water) ${ }^{-1}$ (protoplasts in $0.6 \mathrm{M}$ $\mathrm{KCl}$ ) and, after $5 \mathrm{~min}$ treatment, excess brightener was removed by centrifugation. The cells were observed in a photomicroscope Zeiss III equipped with the UV filter no. 487702 (excitation line $365 / 366 \mathrm{~nm}$ ).

Electron microscopy. Material for electron microscopy was placed on grids coated with Formvar films. After air drying, the specimen was stained with $1 \%(\mathrm{w} / \mathrm{v})$ sodium phosphotungstate and examined in a Jeol $100 \mathrm{~S}$ microscope.

\section{RESULTS}

Microscopic observation of S. cerevisiae and C. albicans with calcofluor

When actively growing cells of $S$. cerevisiae and blastospores of $C$. albicans were observed in the presence of calcofluor with a UV microscope, fluorescence was located mainly in the birth and bud scars and in the cross-wall between mother and bud cells. (Fig. $1 a$ ). But, if actively growing cultures were supplemented with brightener, an increasing deposition of fluorescing material was detected at specific areas in isolated cells (Fig. 1 $b, d$ ) and at the neck between bud and mother cells (Fig. 1c). In some preparations, the brightener was found only in the mother cell, in others in both mother and bud cells. In the former, the buds probably were not physiologically independent of the mother cell. The regions of fluorescence in isolated cells may indicate the location where new buds were about to be formed, but the process had been inhibited by the brightener.

When $C$. albicans growing in a mycelial form was observed in the presence of calcofluor, the brightener was mainly found in the cross-wall and also in the apical areas where hyphal extension took place. (Fig. $1 e$ ). If the hyphae were growing in the presence of calcofluor, a gradually enlarging area of fluorescence corresponding to the cross-wall appeared on incubation, showing that an altered pattern of deposition of calcofluor-absorbing material took place in mycelial cells (Fig. $1 f, g$ ).

Effect of calcofluor on protein synthesis and on the formation of structural components of the wall

The effect of the brightener on synthesis of protein and structural polymers of the wall was dependent on its concentration in the culture medium. No effect was detected at $50 \mu \mathrm{g} \mathrm{ml}^{-1}$, but protein synthesis was partially inhibited at higher concentrations. In the presence of $500 \mu \mathrm{g} \mathrm{ml}^{-1}$, a marked decrease in formation of structural (glucan + chitin) polymers was detected in $S$. cerevisiae $(40 \%)$ and $C$. albicans $(80 \%)$ (data not shown). Similar results were obtained when protoplasts were used instead of cells.

\section{Microscopic observations of regenerating protoplasts after treatment with calcofluor}

Synthesis and deposition of structural components by protoplasts were followed either in the presence or absence of calcofluor in the regeneration medium. The initial step in regeneration of C. albicans protoplasts was the formation of a layer of microfibrillar components. This was deduced from the fact that naked protoplasts were initially unable to absorb the brightener (they did not show fluorescence after the addition of calcofluor; Fig. 2a). Formation of abnormal tubes or hyphae was followed by either a chain of normal budding cells or irregular polymorphic structures. These were the two main routes for regenerating protoplasts. It is important to 


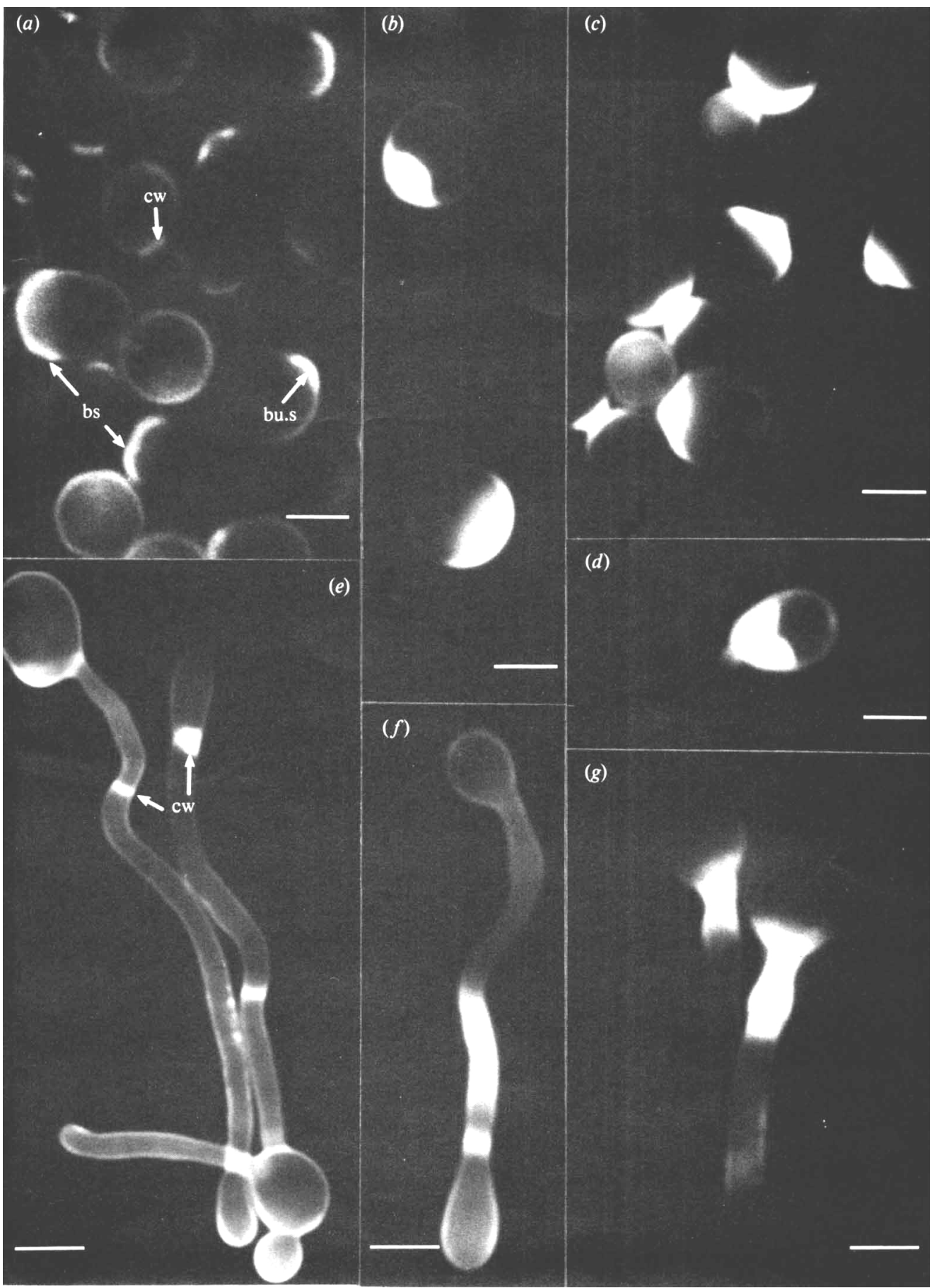

Fig. 1. Fluorescence micrographs of Saccharomyces cerevisiae $(a, b, c)$ and Candida albicans $(d, e, f, g)$. Calcofluor $\left(50 \mu \mathrm{g} \mathrm{ml}^{-1}\right)$ was added to the incubation medium at time zero $(a, e)$, or $2 \mathrm{~h}$ before observations were made $(b, c, d, f, g)$. cw, Cross-wall; bs, birth scar; bu.s., budding scar. The bar markers represent $2 \mu \mathrm{m}$.

emphasize that the fluorescence was more intense at the point of the hyphal emergence and in other specific locations, suggesting that polarization of chitin formation also occurs in reverting protoplasts (Fig. $2 b, c, d$ ). 


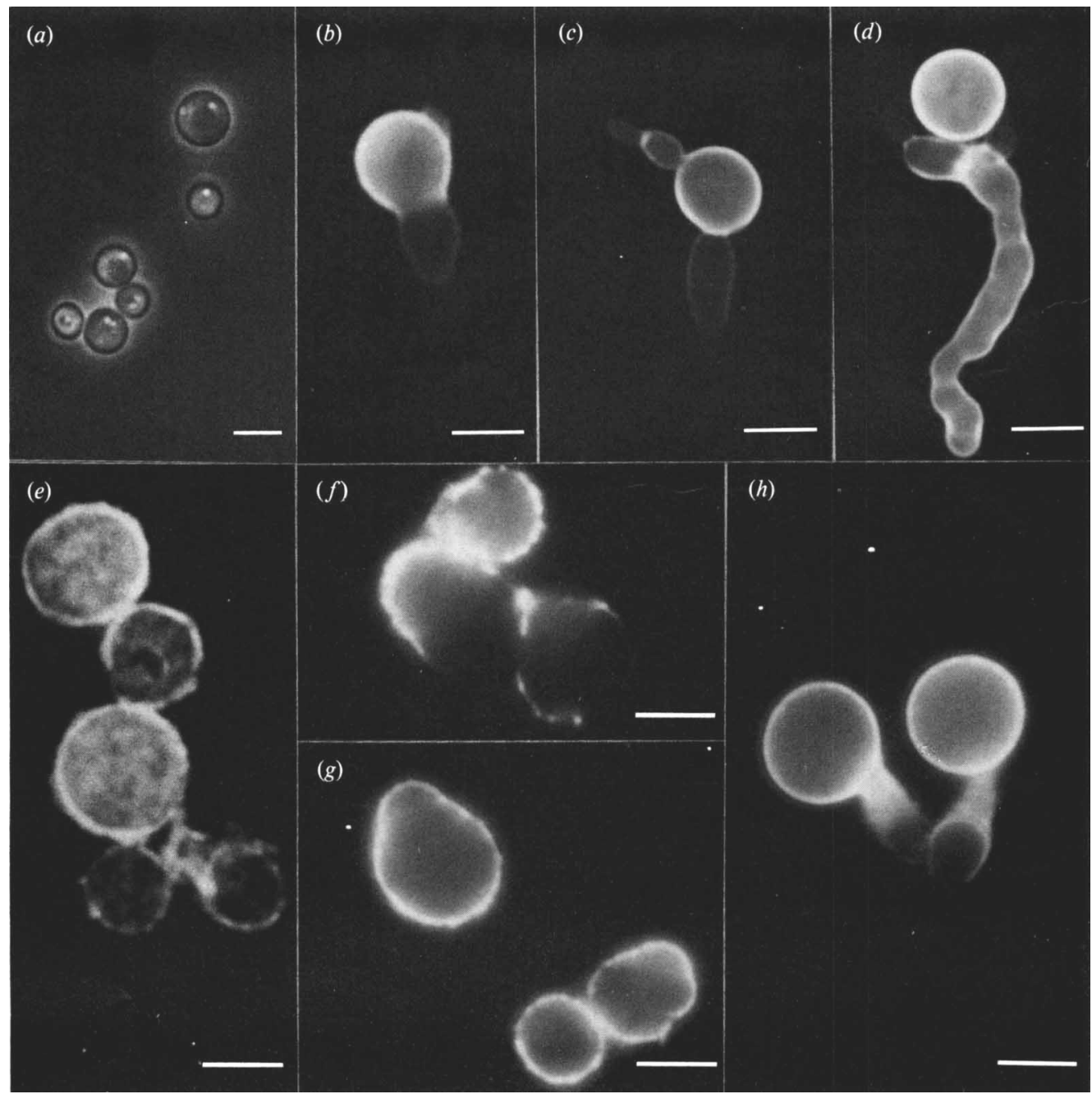

Fig. 2. Phase contrast micrographs of Candida albicans protoplasts (a). Fluorescence micrographs of $C$. albicans regenerating protoplasts after $1 \mathrm{~h}(b, e), 2 \mathrm{~h}(c, f, g)$ and $4 \mathrm{~h}(d, h)$ incubation in the medium of Lee et al. (1975) supplemented with $0.6 \mathrm{M}-\mathrm{KCl}$. Calcofluor $\left(50 \mu \mathrm{g} \mathrm{ml}^{-1}\right)$ was added to the incubation medium at time zero $(b, c, d)$ or $1 \mathrm{~h}(e), 2 \mathrm{~h}(f, g)$ and $4 \mathrm{~h}(h)$ before observations were made. The bar markers represent $2 \mu \mathrm{m}$.

When the regeneration process was studied in protoplasts incubated in the presence of calcofluor, formation of absorbing polymers took place. Initially, small areas developed on the plasma membrane showing fluorescence (Fig. 2e). These areas increased in size gradually and fluorescence was finally found uniformly distributed all over the protoplasts' surface. Reversion of protoplasts in the presence of calcofluor resulted in localized regions showing fluorescence while, in the absence of calcofluor, fluorescence was evenly distributed. The brightener was also found at some distance from the protoplast, appearing as a halo of calcofluor-absorbing material surrounding the protoplast and the abnormal structures formed during the incubation period (Fig. $2 f, g, h$ ).

When incubation of protoplasts was carried out in the absence of calcofluor, a mesh of ribbons covered their surface (Fig. $3 a$ ), which seemed to have been built by parallel arrays of microfibrils. However, if incubation of protoplasts was carried out in the presence of calcofluor, the ribbon assembly seemed to have been disturbed (Fig. $3 b$ ) and single fibrils or aggregates of a few fibrils were observed at higher magnification, indicating a low degree of crystallinity (Fig. $3 c)$. 

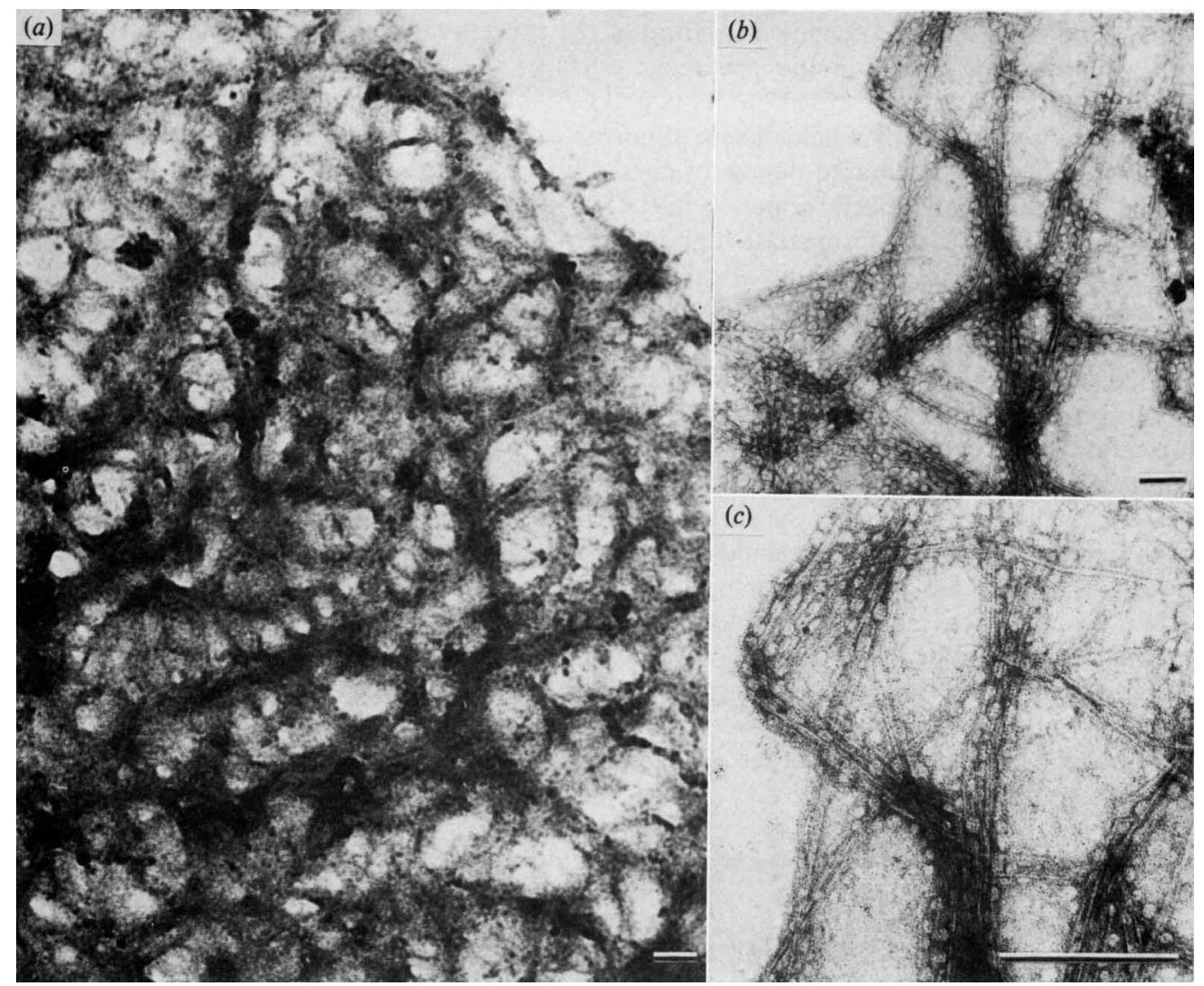

Fig. 3. Transmission electron micrographs of negatively stained preparations of the microfibrillar network synthesized by Candida albicans protoplasts cultured in the medium of Lee et al. (1975) in the absence $(a)$ and presence $(b, c)$ of $50 \mu \mathrm{g}$ calcofluor white $\mathrm{ml}^{-1}$. The bar markers represent $10 \mu \mathrm{m}$.

\section{DISCUSSION}

Calcofluor and other fluorescent brighteners bind to $\beta$-linked fibrillar polymers such as cellulose and chitin (Maeda \& Ishida, 1967). These compounds have therefore been used as vital stains in localization of both cellulose (Haigler et al., 1980) and chitin (Herth, 1980; Peberdy \& Buckley, 1973).

The specificity of calcofluor in binding to yeast chitin was deduced from the following facts: (i) accumulation of fluorescence took place in $S$. cerevisiae almost exclusively at the birth and bud scars and in the cross-wall that separates dividing cells; and (ii) protoplasts did not show fluorescence when incubated under conditions such that formation of $\beta$-glucan occurred, but protein and chitin synthesis were blocked, as in the presence of cycloheximide (unpublished observations). These results indicate that calcofluor may be used as a specific chitin stain in yeast to obtain information on mechanisms of formation and assembly of wall polymers.

When yeast cells were grown in the presence of calcofluor, the cell cycle did not progress and the cells apparently continued to accumulate larger amounts of chitin. Accumulated chitin present at the neck of dividing cells probably corresponded to the location where a disc of chitin is formed before physical separation of the daughter cells occurs (Cabib et al., 1974). In the presence of calcofluor, both formation of the chitin ring and disc were disturbed, and the morphogenetic process halted.

A clearer picture of the calcofluor effect on wall formation may be deduced from the observation of $C$. albicans protoplasts incubated in the presence of the brightener both with 
optical and electron microscopes. Initiation of the process took place with the appearance of chitin-synthesizing areas on the protoplast surface; these areas enlarged until confluence occurred with continued incubation. This process finally gave rise to cell-like structures and aberrant tubes covered by a halo. These abnormal structures seemed to be formed by arrays of chitin fibrils with a decreased degree of crystallinity.

The mechanism(s) of fibril formation in the yeast wall is unknown, but two hypotheses have been proposed: (i) the 'intermediate high-polymer' hypothesis (Colvin, 1964) suggests that the microfibrillar lattice of wall polymers is the result of a two-step process involving polymerization of monomers followed by assembly of the polymers to give rise to microfibrils; (ii) the "endsynthesis' hypothesis proposes that polymerization and crystallization are simultaneous events carried out by an enzymic complex localized in the cell membrane.

Our results suggest that calcofluor did not block synthesis of chitin, but probably inhibited formation of the crystalline lattice by competing for the hydrogen-bonding sites in the nascent polymer. In this situation, the chitin chains may assume a random disposition giving rise to a halo-like structure that covers the surface of the protoplasts. A similar effect of calcofluor has been suggested in formation of cellulose by Acetobacter xylinum (Haigler et al., 1980) and chitin by Poterioochromonas stipitata (Herth, 1980).

We postulate that chitin subunits are polymerized by an enzymic system localized in the plasmalemma (Durán \& Cabib, 1978) associated spontaneously and progressively by formation of hydrogen bonds to give rise to nascent microfibrils. We cannot rule out the possibility that the process of fibril formation might take place with the participation of a 'zippering enzyme' that would be inhibited by the brightener, although this enzyme has never been found in other fungal or plant systems.

This work was supported by the Spanish Comision Asesora de Investigación Científica y Técnica (grant nos 4593-1980 and 120-1982).

\section{REFERENCES}

Cabib, E., Ulane, R. \& Bowers, B. (1974). A molecular model for morphogenesis: the primary septum of yeast. In Current Topics in Cellular Regulation, vol. VIII, pp. 1-32. Edited by B. L. Horecker \& E. R. Stadman. London \& New York: Academic Press.

Colvin, J. R. (1964). The biosynthesis of cellulose. In The Formation of Wood in Forest Trees, pp. 189-201. Edited by M. H. Zimmermann. New York: Academic Press.

Duran, A. \& CABIB, E. (1978). Solubilization and partial purification of yeast chitin synthase. Confirmation of the zymogenic nature of the enzyme. Journal of Biological Chemistry 253, 125-133.

EDDY, A. A. \& WoOdhEAD, J. S. (1968). An alkaliinsoluble glucan fraction from the cell walls of the yeast Saccharomyces carlsbergensis. FEBS Letters 1 , 67-68.

Elorza, M. V., Lostau, C. M., Villanueva, J. R. \& SENTANDREU, R. (1976). Cell wall synthesis regulation in Saccharomyces cerevisiae: effect of RNA and protein inhibition. Biochimica et biophysica acta $\mathbf{4 5 4}$, 263-272.

Haigler, C., Brown, JR, R. M. \& Benziman, M. (1980). Calcofluor white ST alters the in vivo assembly of cellulose microfibrils. Science 210, 903906.

HerTh, H. (1980). Calcofluor white and congo red inhibit chitin microfibril assembly of Poterio- ochromonas: evidence for a gap between polymerization and microfibril formation. Journal of Cell Biology 87, 442-450.

Lee, K. L., Buckley, M. R. \& Campbell, C. (1975). An amino acid liquid synthetic medium for development of mycelial and yeast forms of Candida albicans. Sabouraudia 13, 148-153.

MAEDA, H. \& IsHIDA, L. (1967). Specificity of binding of hexapyranosyl polysaccharides with fluorescent brighteners. Journal of Biochemistry 62, 276-278.

Novick, P., Ferro, S. \& Schekman, R. (1981). Order of events in the yeast secretory pathway. Cell 25, 461-469.

Peberdy, J. F. \& Buckley, C. E. (1973). Adsorption of fluorescent brighteners by regenerating protoplasts of Aspergillus nidulans. Journal of General Microbiology 74, 281-288.

Ruiz-Herrera, J., Sing, V. O., van der Woude, W. J. \& Bartnicki-Garcia, S. (1975). Microfibril assembly by granules of chitin synthase. Proceedings of the National Academy of Sciences of the United States of America 72, 2706-2710.

SlOAT, B. F. \& Pringle, J. R. (1978). A mutant of yeast defective in cellular morphogenesis. Science $\mathbf{2 0 0}$, 1171-1173.

Sloat, B. F., Adams, A. \& Pringle, J. R. (1981). Roles of the cdc 24 gene product in cellular morphogenesis during the Saccharomyces cerevisiae cell cycle. Journal of Cell Biology 89, 395-405. 